\title{
AVALIAÇÃO FÍSICO-gUIMICA DE ÁGUAS MINERAIS COMERCIALIZADAS NA REGIÃO DE CAMPINAS, SP ${ }^{1}$
}

\author{
Marcelo A. MORGANO ${ }^{2, *}$, Ana Cláudia SCHATTI ${ }^{2}$, Hercília A. ENRIQUES ${ }^{2}$, Dilza M.B. MANTOVANI ${ }^{2}$
}

\section{RESUMO}

Com o objetivo de avaliar a qualidade de águas minerais, foram analisadas amostras, adquiridas em supermercados, de oito fontes localizadas no Estado de São Paulo entre agosto de 1999 e maio de 2000, com coletas trimestrais. Os resultados das análises fisicoquímicas mostraram que as amostras possuiam, em sua maioria, composição química dentro dos limites permitidos pela legislação brasileira, exceto quatro amostras, que apresentaram resultados na análise de cor acima de 5 unidades PtCo, uma amostra com pH fora da faixa recomendada de 4,0 a 9,0 e uma amostra com alcalinidade em hidróxidos em duas épocas de análise. Os resultados obtidos para as diferentes amostras variaram de acordo com as seguintes faixas: $\mathrm{pH}$ de 6,39 a 9,92 , alcalinidade de 0,0 a $189,1 \mathrm{mg} \mathrm{L}^{-1}$ de bicarbonato, dureza de 0,00 a $136,00 \mathrm{mg} \mathrm{L}^{-1}$ de carbonato de cálcio, cor de 0,0 a 9,0 unidades de PtCo, turbidez de 0,00 a 2,00 FTU de turvação, sulfato de 0,00 a $55,67 \mathrm{mg} \mathrm{L}^{-1}$, sulfeto de 0,00 a $0,002 \mathrm{mg} \mathrm{L}^{-1}$, cloreto de 0,00 a $23,53 \mathrm{mg} \mathrm{L}^{-1}$, fluoreto de 0,02 a $0,8 \mathrm{mg} \mathrm{L}^{-1}$, alumínio de 0,01 a $0,09 \mathrm{mg} \mathrm{L}^{-1}$, bário de 0,00 a $0,26 \mathrm{mg} \mathrm{L}^{-1}$, cálcio de 0,53 a 28,35mg L-1, crômio de 0,00 a $0,01 \mathrm{mg} \mathrm{L}^{-1}$, ferro de 0,00 a 0,03 $\mathrm{mg} \mathrm{L}^{-1}$, potássio de 0,39 a 3,27 $\mathrm{mg} \mathrm{L}^{-1}$, sódio de 0,53 a $97,29 \mathrm{mg} \mathrm{L} 1$, magnésio de 0,00 a $9,10 \mathrm{mg} \mathrm{L}^{-1}$, manganês de 0,00 a $0,05 \mathrm{mg} \mathrm{L}^{-1}$, zinco de 0,00 a $0,03 \mathrm{mg} \mathrm{L}^{-1}$, chumbo de 0,00 a $0,01 \mathrm{mg} \mathrm{L}^{-1}$ e litio de 0,00 a $0,02 \mathrm{mg} \mathrm{L}^{-1}$. Os elementos cádmio, cobre e cobalto apresentaram-se abaixo dos limites de detecção dos métodos.

Palavras-chave: águas minerais; parâmetros físico-químicos; oligoelementos; espectrometria de emissão de plasma.

\section{SUMMARY}

PHYSICAL-CHEMICAL EVALUATION OF MINERAL WATERS MARKETED IN CAMPINAS, SP. Samples from eight different sources of mineral water from São Paulo State were purchased from August 1999 to May 2000 in order to evaluate the physical-chemical quality. In general, most samples were within the limits permited by the Brazilian legislation, except for four samples which showed color results above 5 PtCo units, one sample with $\mathrm{pH}$ outside the recommended range ( $\mathrm{pH} 4$ to 9) and one sample which presented hydroxide alkalinity in two different periods of sampling. The results varied according to the following ranges: $\mathrm{pH}$ from 6.39 to 9.92 , alkalinity from 0.00 to $189.10 \mathrm{mg} \mathrm{L}^{-1} \mathrm{de} \mathrm{HCO}_{3}^{-1}$, hardness from 0.00 to $136.00 \mathrm{mg} \mathrm{L}^{-1}$ carbonate of calcium, color from 0.0 to 9.0 units PtCo, turbidity from 0.00 to 2.00 FTU of turbidity, sulphate from 0.00 to $55.67 \mathrm{mg} \mathrm{L}^{-1}$, sulfide from 0.00 to $0.002 \mathrm{mg} \mathrm{L}^{-1}$, chloride from 0.00 to $23.53 \mathrm{mg} \mathrm{L}$, fluoride de 0.02 a $0.8 \mathrm{mg} \mathrm{L}^{-1}$ aluminum from 0.01 to $0.09 \mathrm{mg} \mathrm{L}^{-1}$, barium from 0.00 to $0.26 \mathrm{mg} \mathrm{L}^{-1}$, calcium from 0.53 to $28.35 \mathrm{mg} \mathrm{L} \mathrm{L}^{-1}$, chromium from 0.00 to $0.01 \mathrm{mg} \mathrm{L}^{-1}$, iron from 0.00 to $0.03 \mathrm{mg} \mathrm{L}^{-1}$, potassium from 0.39 to $3.27 \mathrm{mg} \mathrm{L}^{-1}$, sodium from 0.53 to $97.29 \mathrm{mg} \mathrm{L} 1$, magnesium from 0.00 to $9.10 \mathrm{mg} \mathrm{L}^{-1}$, manganese from 0.00 to $0.05 \mathrm{mg} \mathrm{L}^{-1}$, zinc from 0.00 to $0.03 \mathrm{mg} \mathrm{L}^{-1}$, lead from 0.00 to $0.01 \mathrm{mg} \mathrm{L}^{-1}$ and lithium from 0.00 to $0.02 \mathrm{mg}$ $\mathrm{L}^{-1}$. The elements cadmium, copper and cobalt were below the limits of detection for the analytical methods.

Keywords: mineral water; physical-chemical parameters; oligoelements; atomic emission spectrometry.

\section{1 - INTRODUÇÃO}

De acordo com a Resolução RDC no 54 de 15 de junho de 2000 da Agência Nacional de Vigilância Sanitária [4], a água mineral é caracterizada pelo conteúdo definido e constante de sais minerais, pela presença de oligoelementos e outros constituintes. Esta resolução também estabelece os padrões de identidade e qualidade para a água mineral natural no Brasil.

As águas minerais são classificadas quanto a sua composição química conforme o elemento predominante [5] e sua composição varia de acordo com as rochas e terrenos pelos quais a mesma passou enquanto infiltravase no solo, podendo, também, ter contribuições das águas meteóricas, clima e biota.

No Brasil, o consumo de água mineral vem crescendo continuamente, principalmente nas regiões Sudeste e Nordeste. O volume de produção que era de 1 bilhão e meio, em 1995, passou para mais de 2 bilhões de litros,

\footnotetext{
Recebido para publicação em 29/08/2000. Aceito para publicação em $07 / 02 / 2002$.

2. Ital (Instituto de Tecnologia de Alimentos). Centro de Química. Av. Brasil, 2880 - Jd. Chapadão - CEP 13073-001 - Cx. Postal $139-$ Campinas-SP.

* A quem a correspondência deve ser enviada.
}

em 1997 [14]. O Estado de São Paulo é o maior produtor do País de água mineral e potável de mesa [1].

O crescimento no consumo de águas minerais está relacionado principalmente à poluição dos rios que abastecem as grandes cidades e aos efeitos medicinais benéficos para a saúde que os consumidores acreditam que as águas minerais possam ter. Assim, o conhecimento das características físico-químicas das águas minerais é muito importante para a garantia da saúde da população [9].

Uma das formas mais perigosas de contaminação das águas de consumo humano é pelos metais pesados. A ingestão destes metais pode causar diversos problemas à saúde humana, como distúrbios gastrointestinais até disfunção mental com degeneração do sistema nervoso central [3].

Existem poucas referências sobre a qualidade de águas minerais brasileiras. NISHIHARA, ALABURDA, MAIO [12] analisaram amostras de 12 fontes minerais da Região Metropolitana de São Paulo quanto a parâmetros físico-químicos estabelecidos pela legislação vigente obtendo valores aceitáveis, exceto para uma fonte que apresentou indices de manganês e nitrato acima do previsto. 
PEDRO et al. [13] determinaram a presença de treze metais (Al, As, Ca, Cd, Co, Cr, Cu, Fe, Mg, Mn, Ni, Pb e $\mathrm{Zn}$ ) em trinta e oito amostras de água mineral de fontes da região de Campinas, SP e de nove amostras envazadas com diferentes tipos de embalagens encontrando baixos teores dos elementos pesquisados, demonstrando que as águas minerais da região estudada estavam adequadas para o consumo humano quanto à presença de metais. As análises foram efetuadas pela técnica de espectrometria de emissão atômica com fonte de excitação de plasma de argônio induzido (ICP-AES).

Garantir fontes de água de boa qualidade é uma tarefa dificil e estando a qualidade da água diretamente ligada à saúde humana, determinar e controlar as suas características físico-químicas se faz necessário para garantir a segurança quanto ao consumo de água pela população. Assim, este trabalho teve como objetivo avaliar as características físico-químicas de águas minerais procedentes de fontes localizadas no Estado de São Paulo, cujas águas minerais encontram-se disponiveis comercialmente na região de Campinas, SP.

\section{2 - MATERIAL E MÉTODOS}

\section{1 - Amostras}

Foram coletadas amostras de águas minerais em supermercados da região de Campinas em três épocas (outubro/1999, dezembro/1999, março/2000). Oito marcas foram selecionadas e duas garrafas de cada uma foram analisadas. Os tipos de amostras estão apresentados na Tabela 1.

TABELA 1. Identificação das amostras de águas minerais

\begin{tabular}{cl}
\hline Amostra & \multicolumn{1}{c}{ Classificação } \\
\hline YC & Fluoretada \\
AS & Radioativa na fonte \\
SB & Fluoretada fracamente radioativa na fonte \\
SJ & Fracamente radioativa na fonte \\
NS & Fracamente radioativa na fonte \\
SP & Fluoretada fracamente radioativa na fonte \\
IB & Alcalina bicarbonatada, fluoretada, vanádica \\
L3 & Fluoretada radioativa na fonte \\
\hline \hline
\end{tabular}

\section{2 - Parâmetros analisados e métodos utilizados}

Os métodos utilizados nas determinações dos parâmetros físico-químicos das águas minerais estão apresentados resumidamente na Tabela 2. Para as determinações dos teores dos ânions foi utilizado um espectrofotômetro HACH, modelo DR-2000 (Colorado, USA) [8]. Para os cátions, após digestão e concentração das amostras, segundo o método da AOAC [7] utilizou-se o equipamento de ICP-AES BAIRD, modelo ICP 2000 (Massachusetts, USA).

As Tabelas 3 e 4 apresentam as condições de operação, limites de detecção e os parâmetros de curva analítica das determinações dos metais.
TABELA 2. Parâmetros analisados e métodos utilizados

\begin{tabular}{|c|c|c|}
\hline Parâmetro & Método & Descrição \\
\hline Alcalinidade & $\begin{array}{l}\text { Volumetria de } \\
\text { neutralização [11] }\end{array}$ & $\begin{array}{l}\text { Reagente: } \mathrm{HCl} \text {; Indicadores fenolftaleína } \\
\text { e metilorange }\end{array}$ \\
\hline Dureza & $\begin{array}{l}\text { Volumetria de } \\
\text { complexação [10] }\end{array}$ & $\begin{array}{l}\text { Reagente: EDTA } \\
\text { Indicador: Preto de Eriocromo T }\end{array}$ \\
\hline $\mathrm{pH}$ & Potenciométrico [10] & - \\
\hline Cloreto & Espectrofotometria [8] & $\begin{array}{l}\lambda=455 \mathrm{~nm} ; \text { reação com tiocianato de } \\
\text { mercúrio e ferro }\end{array}$ \\
\hline Sulfato & Espectrofotometria [8] & $\lambda=450 \mathrm{~nm}$; reação com íons bário \\
\hline Sulfeto & Espectrofotometria [8] & $\begin{array}{l}\lambda=665 \mathrm{~nm} \text {; reação com } \\
\mathrm{N}, \mathrm{N} \text {-dimetil-p-fenilenodiamino-oxalato }\end{array}$ \\
\hline Fluoreto & Espectrofotometria [8] & $\lambda=580 \mathrm{~nm} ;$ reagente de "Spands" \\
\hline Cor aparente & Espectrofotometria [8] & $\lambda=455 \mathrm{~nm}$ \\
\hline Turbidez & Espectrofotometria [8] & $\lambda=450 \mathrm{~nm}$ \\
\hline
\end{tabular}

TABELA 3. Condições de operação do espectrômetro

\begin{tabular}{lc}
\hline \multicolumn{1}{c}{ Parâmetro } & Condição de operação \\
\hline Potência do Plasma & $1,1 \mathrm{Kw}$ \\
Gás refrigerante (Ar) & $6,5 \mathrm{~L} / \mathrm{min}$ \\
Gás auxiliar (Ar) & $6,5 \mathrm{~L} / \mathrm{min}$ \\
Vazão da amostra & $2,1 \mathrm{~mL} / \mathrm{min}$ \\
Altura de observação vertical & $19 \mathrm{~mm}^{\text {a }}$ \\
Pressão do nebulizador $^{\text {b }}$ & $3 \mathrm{bar}$ \\
\hline \hline
\end{tabular}

a acima da bobina de indução; ${ }^{b}$ nebulizador pneumático concêntrico.

TABELA 4. Metais determinados, comprimentos de onda $(\lambda)$, limites de detecção (L.D.) e faixa de trabalho das curvas analiticas

\begin{tabular}{lccc}
\hline Metal & $\begin{array}{c}\lambda \\
(\mathbf{n m})\end{array}$ & $\begin{array}{c}\text { L.D. } \\
\left(\mathbf{m g ~ L}^{-1}\right)\end{array}$ & $\begin{array}{c}\text { Faixa de trabalho } \\
\text { da curva (mg L }\end{array}$ \\
\hline Alumínio & 308,21 & 0,012 & 0,025 a 4 \\
Bário & 493,41 & 0,001 & 0,025 a 4 \\
Cobre & 324,75 & 0,003 & 0,005 a 0,5 \\
Ferro & 259,94 & 0,004 & 0,005 a 0,5 \\
Lítio & 670,78 & 0,005 & 0,005 a 0,5 \\
Manganês & 257,67 & 0,0003 & 0,005 a 0,5 \\
Zinco & 213,86 & 0,002 & 0,005 a 0,5 \\
Cromo & 267,71 & 0,002 & 0,005 a 0,5 \\
Cobalto & 228,62 & 0,002 & 0,005 a 0,5 \\
Chumbo & 220,35 & 0,005 & 0,005 a 0,5 \\
Cádmio & 226,50 & 0,001 & 0,005 a 0,5 \\
Cálcio & 317,93 & 0,013 & 1 a 100 \\
Sódio & 589,59 & 0,029 & 1 a 100 \\
Magnésio & 279,08 & 0,041 & 1 a 100 \\
Potássio & 766,49 & 0,171 & 1 a 100 \\
\hline
\end{tabular}

L.D. = limite de detecção ( 3 vezes o desvio padrão de 10 leituras de branco).

\section{3 - RESUlTADOS E DISCUSSÃO}

Realizou-se a avaliação dos métodos analíticos verificando a repetibilidade, e os niveis de recuperação para uma amostra de água mineral. A repetibilidade foi avaliada pelo coeficiente de variação $(\mathrm{CV})$ determinado através da preparação de 10 repetições analiticas para os cátions e 5 repetições analiticas de uma das amos- 
tras de água mineral. Para a avaliação do nivel de recuperação dos minerais, uma amostra foi fortificada com os metais $\mathrm{Al}, \mathrm{Ba}, \mathrm{Cu}, \mathrm{Fe}, \mathrm{Li}, \mathrm{Mn}, \mathrm{Zn}, \mathrm{Cr}, \mathrm{Co}, \mathrm{Pb}, \mathrm{Cd}, \mathrm{Ca}$, $\mathrm{Na}, \mathrm{Mg}$ e K. As Tabelas 5 e 6 apresentam os resultados obtidos.

As análises realizadas no espectrofotômetro DR/ 2000 apresentaram boa repetibilidade com exceção da análise de sulfeto que obteve um alto coeficiente de variação, porém esse fato pode ser devido aos baixos niveis de sulfeto encontrados nas amostras analisadas.

TABELA 5. Avaliação da repetibilidade para a determinação dos ânions cloreto, sulfato, fluoreto e sulfeto em amostra de água mineral

\begin{tabular}{lcc}
\hline \multicolumn{1}{c}{ Determinação } & $\begin{array}{c}\text { Média } \\
\left(\mathbf{m g ~ L}^{-1} \mathbf{)}\right.\end{array}$ & $\begin{array}{c}\mathbf{C V}^{*} \\
\mathbf{( \% )}\end{array}$ \\
\hline Cloreto & 1,00 & 0,00 \\
Sulfato & 4,00 & 0,00 \\
Fluoreto & 0,64 & 1,30 \\
Sulfeto & 0,006 & 34,80 \\
\hline
\end{tabular}

*Coeficiente de variação referente a 5 repetições analíticas.

TABELA 6. Avaliação da repetibilidade e níveis de recuperação para a determinação dos metais em amostra de água mineral

\begin{tabular}{lccc}
\hline Metal & $\begin{array}{c}\text { Média } \\
\left(\mathbf{m g ~ L}^{-1} \mathbf{)}\right.\end{array}$ & $\begin{array}{l}\mathbf{C V}^{*} \\
\mathbf{( \% )}\end{array}$ & $\begin{array}{c}\text { Niveis de Recuperação } \\
(\mathbf{\%})\end{array}$ \\
\hline Alumínio & 0,59 & 14,2 & 117 \\
Bário & 0,77 & 1,3 & 99 \\
Cobre & 0,06 & 2,0 & 137 \\
Ferro & 0,04 & 2,6 & 109 \\
Lítio & 0,06 & 1,9 & 100 \\
Manganês & 0,06 & 1,8 & 106 \\
Zinco & 0,08 & 2,6 & 94 \\
Crômio & 0,06 & 2,5 & 108 \\
Cobalto & 0,05 & 2,4 & 104 \\
Chumbo & 0,03 & 5,1 & 109 \\
Cádmio & 0,05 & 3,2 & 103 \\
Cálcio & 40,21 & 1,1 & 106 \\
Sódio & 45,16 & 3,3 & 102 \\
Magnésio & 31,01 & 0,8 & 101 \\
Potássio & 32,43 & 3,4 & 98 \\
\hline
\end{tabular}

${ }^{\text {*} C o e f i c i e n t e ~ d e ~ v a r i a c ̧ a ̃ o ~ r e f e r e n t e ~ a ~} 10$ repetições analíticas.

A maior variação na determinação de metais ocorreu na determinação de alumínio $(14,2 \%)$ e o menor na de magnésio $(0,8 \%)$.

O nivel de recuperação obtido para o elemento cobre está acima do recomendado por CARDONE [6], ou seja, 75 a $125 \%$, entretanto, o elemento não foi detectado em nenhuma das amostras analisadas. Para os demais elementos, os niveis de recuperação encontrados estão de acordo com a faixa recomendada por CARDONE [6].

Nas Tabelas 7 e 8 estão descritos os resultados obtidos na caracterização físico-química das águas mine- rais provenientes das oito fontes analisadas durante as três épocas de análises. Verificando os valores médios obtidos para todas as águas analisadas, observa-se que o ín cloreto teve uma diminuição da primeira para a terceira época $\left(4,47\right.$ para $\left.0,56 \mathrm{mg} \mathrm{L}^{-1}\right)$. O ion bicarbonato aumentou na segunda época, mantendo-se constante na primeira e terceira épocas. Os elementos cálcio e magnésio também apresentaram aumento na segunda época e constante nas demais. Os demais parâmetros mostraram resultados médios próximos em todas as três épocas de análise.

TABELA 7. Valores máximos, mínimos, média e desvio padrão (DP) das análises físico-químicas

\begin{tabular}{|c|c|c|c|c|c|c|c|c|c|}
\hline & $\mathrm{pH}$ & $\begin{array}{l}\text { Sulfato } \\
(\mathrm{mg} / \mathrm{L})\end{array}$ & $\begin{array}{l}\text { Cloreto } \\
(\mathrm{mg} / \mathrm{L})\end{array}$ & $\begin{array}{l}\text { Dureza } \\
(\mathrm{mg} / \mathrm{L}) \\
\end{array}$ & $\begin{array}{c}\text { Bicarbonato } \\
(\mathrm{mg} / \mathrm{L})\end{array}$ & $\begin{array}{r}\mathrm{Cor}^{*} \\
(\mathrm{PtCo})\end{array}$ & $\begin{array}{c}\text { Turbidez } \\
\text { (FTU) }\end{array}$ & $\begin{array}{c}\text { Fluoreto } \\
(\mathrm{mg} / \mathrm{L})\end{array}$ & $\begin{array}{l}\text { Sulfeto } \\
(\mathrm{mg} / \mathrm{L})\end{array}$ \\
\hline \multirow{4}{*}{ 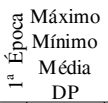 } & 9,83 & 55,67 & 23,53 & 107,33 & 115,89 & 9,00 & 1,00 & \multirow[t]{4}{*}{$\mathrm{n} / \mathrm{a}$} & 0,002 \\
\hline & 6,84 & 0,00 & 0,00 & 0,00 & 0,00 & 0,00 & 0,00 & & 0,000 \\
\hline & 7,72 & 9,82 & 4,47 & 55,56 & 75,52 & 2,18 & 0,07 & & 0,001 \\
\hline & 0,88 & 19,02 & 7,82 & 37,44 & 39,86 & 2,78 & 0,26 & & 0,001 \\
\hline \multirow{4}{*}{ 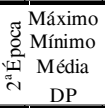 } & 9,92 & 55,00 & 11,47 & 136,00 & 189,10 & 4,00 & 1,00 & \multirow[t]{4}{*}{ n/a } & \multirow[t]{4}{*}{$\mathrm{n} / \mathrm{a}$} \\
\hline & 7,13 & 0,00 & 0,46 & 1,00 & 1,76 & & 0,00 & & \\
\hline & 7,79 & 5,95 & 2,91 & 66,71 & 111,40 & 1,26 & 0,36 & & \\
\hline & 0,87 & 20,06 & 0,46 & 1,00 & 1,76 & 1,15 & 0,00 & & \\
\hline \multirow{4}{*}{ 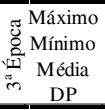 } & 9,88 & 54,67 & 1,90 & 96,67 & 143,35 & 7,00 & 2,00 & 0,80 & \multirow[t]{4}{*}{$\mathrm{n} / \mathrm{a}$} \\
\hline & 6,39 & 0,0 & & 0,00 & & 0,00 & 0,00 & 0,02 & \\
\hline & 7,83 & 7,64 & 0,56 & 47,25 & 77,43 & 4,38 & 1,23 & 0,26 & \\
\hline & 1,07 & 18,76 & 0,77 & 36,34 & 49,33 & 2,69 & 1,01 & 0,27 & \\
\hline \multirow{4}{*}{$\begin{array}{c}\text { Máximo } \\
\text { ్ㅝㅇ Mínimo } \\
\circlearrowleft ّ \text { Média } \\
\text { DP }\end{array}$} & 9,92 & 55,67 & 23,53 & 136,00 & 189,10 & 9,00 & 2,00 & 0,80 & 0,002 \\
\hline & 6,39 & 0,00 & 0,00 & 0,00 & 0,00 & 0,00 & 0,00 & 0,02 & 0,000 \\
\hline & 7,83 & 8,95 & 2,74 & 55,39 & 90,42 & 2,52 & 0,51 & 0,26 & 0,001 \\
\hline & 0,90 & 18,41 & 5,25 & 39,90 & 51,01 & 2,67 & 0,80 & 0,27 & 0,001 \\
\hline
\end{tabular}

TABELA 8. Teores máximos, mínimos, média e desvio padrão (DP) das análises dos metais em amostras de água mineral

\begin{tabular}{|c|c|c|c|c|c|c|c|c|c|c|c|c|}
\hline & & & & Teo & res de $\mathrm{M}$ & Tetais (m & $\mathrm{g} / \mathrm{L})$ & & & & & \\
\hline & $\mathrm{Al}$ & $\mathrm{Ba}$ & $\mathrm{Fe}$ & $\mathrm{Li}$ & $\mathrm{Mn}$ & $\mathrm{Zn}$ & $\mathrm{Cr}$ & $\mathrm{Pb}$ & $\mathrm{Ca}$ & $\mathrm{Na}$ & $\mathrm{Mg}$ & $\mathrm{K}$ \\
\hline Máximo & 0,07 & 0,22 & 0,02 & 0,01 & 0,04 & 0,03 & $<0,005$ & $<0,005$ & 28,35 & 97,29 & 9,10 & 3,27 \\
\hline & 0,0 & & & & & $<0,005$ & & & 53 & 0,71 & 0,01 & ,43 \\
\hline ¿ Méd & 0,03 & 0,0 & 0,0 & & 0,04 & 0,01 & & $<0$, & 12,98 & 25,61 & 4,77 & 1,97 \\
\hline- DP & 0,02 & 0,07 & 0,01 & 0,00 & 0,00 & 0,01 & & & 8,95 & 31,59 & 3,30 & 0,88 \\
\hline ¿ Máx & 0,09 & 0,26 & & 0,0 & & 0,03 & 0,01 & 0,01 & 28,35 & 95,30 & 9,10 & 3,27 \\
\hline ¿̋ Mín & 0,12 & $<0,025$ & $<0,005$ & $<0,005$ & $<0,005$ & & & 0,01 & 0,53 & 1,38 & 0,00 & 0,43 \\
\hline स्ञે Mé & 0,03 & 0,09 & 0,01 & 0,01 & & 0 & 0,01 & 01 & 12,37 & 19,81 & 4,77 & 1,97 \\
\hline$\approx \mathrm{DP}$ & 0,12 & 0,00 & 0,00 & 0,00 & & 0,01 & 0,01 & 0,01 & 8,37 & 32,86 & $\begin{array}{l}3,30 \\
\end{array}$ & 0,88 \\
\hline gáx & 0 & 0,1 & & & & 0,02 & & $<0,0$ & 27,22 & 93,89 & 8,48 & 29 \\
\hline $\mathrm{N}$ & 0 & & & & & & & & & & & ת, \\
\hline $\mathrm{M}$ & 0,03 & 0,0 & 0,0 & 0,01 & & 0,01 & $<0,0$ & & 11,54 & & 4,14 & 2,11 \\
\hline लే DP & 0,01 & 0,04 & 0,01 & 0,00 & 0,02 & 0,01 & & & 9,28 & 33,16 & 3,22 & 0,95 \\
\hline Máxin & 0,09 & 0,26 & & 02 & & 0,03 & & & 28,35 & 97,29 & 9,10 & 3,27 \\
\hline & & & & & & $<0,005$ & & & & & & \\
\hline $\mathrm{M}$ & 0,03 & 0,08 & 0,01 & 0,01 & 0,02 & 0,02 & 0,01 & 0,01 & 12,31 & 24,72 & 4,58 & 2,01 \\
\hline DP & 0,02 & 0,07 & 0,01 & 0,00 & 0,02 & 0,01 & & & 8,85 & 30,92 & 3,21 & 0,8 \\
\hline
\end{tabular}

As Tabelas 9 e 10 apresentam os resultados obtidos para todas as determinações realizadas nas amostras provenientes das diferentes fontes de água mineral.

Apesar de estar declarado no rótulo como água alcalino-bicarbonatada, os valores de $\mathrm{pH}$ obtidos $(9,55$ a 9,92) nas amostras IB foram sempre acima da faixa recomendada pela legislação brasileira que é de 4 a 9 [4]. Este fato foi confirmado na análise de alcalinidade que indicou em duas épocas a presença de íons hidróxido e carbonato. O Ministério da Saúde determina que as águas minerais não devem apresentar alcalinidade em hidróxido [4]. Nas demais águas, os valores de $\mathrm{pH}$ estiveram próximos da neutralidade e dentro da faixa recomendada, 
sendo que a alcalinidade nestas amostras foi devido apenas à presença de íons bicarbonato.

TABELA 9. Valores mínimos, máximos, média e desvio padrão (DP) das análises físico-químicas para cada fonte de água mineral analisada

\begin{tabular}{|c|c|c|c|c|c|c|c|c|c|c|}
\hline \multicolumn{2}{|c|}{ Fonte } & \multirow{2}{*}{$\begin{array}{c}\mathrm{pH} \\
9,55\end{array}$} & \multicolumn{2}{|c|}{$\begin{array}{l}\text { Sulf ato Cloreto } \\
(\mathrm{mg} / \mathrm{L})(\mathrm{mg} / \mathrm{L})\end{array}$} & \multirow{2}{*}{$\begin{array}{r}\begin{array}{r}\text { Dureza } \\
(\mathrm{mg} / \mathrm{L})\end{array} \\
0,00\end{array}$} & \multirow{2}{*}{$\begin{array}{r}\begin{array}{c}\text { Bicarb onato } \\
(\mathrm{mg} / \mathrm{L})\end{array} \\
0,00\end{array}$} & \multirow{2}{*}{$\begin{array}{c}\begin{array}{c}\text { Cor } \\
(\mathrm{PtCo})\end{array} \\
0,00\end{array}$} & \multirow{2}{*}{$\begin{array}{r}\begin{array}{c}\text { Turbidez } \\
\text { (FTU) }\end{array} \\
0,00\end{array}$} & \multirow{2}{*}{$\begin{array}{r}\begin{array}{r}\text { Fuoreto } \\
(\mathrm{mg} / \mathrm{L})\end{array} \\
0,39\end{array}$} & \multirow{2}{*}{$\begin{array}{r}\begin{array}{r}\text { Sulf eto } \\
(\mathrm{mg} / \mathrm{L})\end{array} \\
0,002\end{array}$} \\
\hline & Mínimo & & 44,67 & 1,50 & & & & & & \\
\hline \multirow[t]{4}{*}{ IB } & Máximo & 9,92 & 55,67 & 3,50 & 0,00 & 189,10 & 6,00 & 0,00 & 0,39 & 0,002 \\
\hline & Média & 9,81 & 52,22 & 2,63 & 0,00 & 63,03 & 1,83 & 0,00 & 0,39 & 0,002 \\
\hline & DP & 0,13 & 4,09 & 0,84 & 0,00 & 97,65 & 2,32 & ,00 & 0,00 & 0,000 \\
\hline & Mínimo & 6,84 & 0,00 & 1,10 & 69,33 & 88,45 & 0,00 & 0,00 & 0,14 & 0,000 \\
\hline \multirow[t]{4}{*}{ SJ } & Máximo & 7,41 & 0,00 & 4,10 & 71,67 & 94,55 & 6,00 & 2,00 & 0,15 & 0,000 \\
\hline & Média & 7,18 & 0,00 & 2,77 & 70,26 & 92,52 & 2,00 & 0,67 & 0,15 & 0,000 \\
\hline & DP & 0,26 & 0,00 & 1,33 & 0,77 & 3,15 & 3,10 & 1,03 & 0,01 & 0,000 \\
\hline & Mínimo & & & 0,00 & 14,00 & 97, & 1,00 & $\overline{00}$ & 0,80 & 0,000 \\
\hline \multirow[t]{3}{*}{ YC } & Máximo & 8,01 & 0,00 & 0,70 & 16,60 & 106,75 & 5,00 & 2,00 & 0,80 & 0,000 \\
\hline & Média & 7,66 & & 0,38 & 15,10 & 102,68 & 3,33 & 0,67 & 0,80 & 0,000 \\
\hline & DP & 0,27 & 0,00 & 0,31 & 1,05 & 4,17 & 1,86 & 1,03 & 0,00 & 0,000 \\
\hline & Mínimo & 7,10 & & & 78,67 & 91, & 0,00 &, 00 & 0,14 & 0,000 \\
\hline \multirow[t]{3}{*}{ SB } & Máximo & 8,00 & 0,00 & 0,60 & 96,67 & 143,35 & 6,00 & 2,00 & 0,16 & 0,000 \\
\hline & Média & 7,62 & 0 & 0,23 & 84,80 & 116,07 & 2,33 & 0,67 & 0,15 & 0,000 \\
\hline & DP & 0,41 & 0,00 & 0,29 & 8,94 & 23,29 & 2,88 & 1,03 & 0,01 & 0,000 \\
\hline \multirow{4}{*}{ AS } & Mínin & & & & & & 0,00 & 00 & 0,04 & 0,000 \\
\hline & Máximo & 8,05 & 0,00 & 1,00 & 107,67 & 158,60 & 7,00 & 2,00 & 0,04 & 0,000 \\
\hline & Média & & & 0,33 & & & & 0,67 & 0,04 & \\
\hline & DP & 0,17 & 0,00 & 0,52 & 2,47 & 30,90 & 2,58 & 1,03 & 0,00 & 0,000 \\
\hline & Mínimo & 6,39 & 0,00 & 0,00 & 7,00 & 8,80 & 0,00 & 0,00 & 0,13 & 0,001 \\
\hline \multirow[t]{3}{*}{ L3 } & Máximo & 8,07 & & 1,30 & 48,00 & 61,00 & 2,00 & 1,00 & 0,16 & 0,001 \\
\hline & Média & 7,32 & 0,22 & 0,77 & 44,17 & 54,90 & 0,50 & 0,33 & 0,15 & 0,001 \\
\hline & DP & 0,76 & & 0,61 & 5,5 & & 0,84 &, 52 & 0,02 & 0,000 \\
\hline & Mínim & 7,44 & 15,00 & 11,30 & 106,67 & 69,53 & 0,00 & 0,00 & 0,00 & 0,001 \\
\hline \multirow[t]{4}{*}{ SP } & Máximo & 7,75 & 21,00 & 23,50 & 136,00 & & 5,00 & 1,00 & 0,00 & 0,001 \\
\hline & Média & 7,67 & 17,33 & 17,45 & 121,50 & 82 & 2,00 & 0,50 & & 0,001 \\
\hline & DP & 0,15 & 2,91 & 6,99 & 16,75 & 53,26 & 2,45 & 0,58 & & 0,000 \\
\hline & Míni & & & 1,60 & 16,00 & 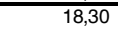 & 0,00 & 0,00 & 0,02 & 0,00 \\
\hline \multirow[t]{3}{*}{ NS } & Máxir & 7,3 & & 2,00 & 25,6 & 20 & 9,00 & 1,00 & 0,02 & 0,00 \\
\hline & Média & 7,17 & 0,33 & 1,83 & 19,78 & 18,98 & 4,33 & 0,67 & 0,02 & 0,00 \\
\hline & DP & 0,20 & 0,58 & 0,21 & 5,17 & 1,17 & 4,51 & 0,58 & 0,00 & 0,00 \\
\hline
\end{tabular}

TABELA 10. Teores mínimos, máximos, média e desvio padrão das análises dos metais para cada fonte de água mineral analisada

\begin{tabular}{|c|c|c|c|c|c|c|c|c|c|c|c|c|c|}
\hline \multirow[b]{2}{*}{ Fonte } & & \multicolumn{11}{|c|}{ Teores de Metais em mg/L } & \multirow{3}{*}{$\mathrm{K}$} \\
\hline & & $\mathrm{AL}$ & $\mathrm{Ba}$ & $\mathrm{Fe}$ & $\mathrm{Li}$ & $\mathrm{Mn}$ & $\mathrm{Zn}$ & $\mathrm{Cr}$ & $\mathrm{Pb}$ & $\mathrm{Ca}$ & $\mathrm{Na}$ & $\mathrm{Mg}$ & \\
\hline & Mínimo & 0,03 & $<0,005$ & 0,02 & $<0,005$ & $<0,005$ & 0,01 & $<0,005$ & $<0,005$ & 0,53 & 93,61 & 0,00 & \\
\hline \multirow[t]{3}{*}{ E } & Máximo & 0,07 & $<0,005$ & 0,03 & $<0,005$ & $<0,005$ & 0,01 & $<0,005$ & $<0,005$ & 0,62 & 97,29 & 0,02 & 0,45 \\
\hline & & 0,04 & $<0,005$ & 0,02 & $<0,005$ & $<0,005$ & 0,01 & $<0,005$ & $<0,005$ & 0,55 & 95,18 & 0,01 & 0,42 \\
\hline & DP & 0,02 & & 0,00 & & & 0,00 & & & 0,04 & 1,67 & 0,01 & 0,03 \\
\hline & Mínimo & 0,03 & 0,08 & $<0,005$ & 0,01 & $<0,005$ & 0,00 & $<0,005$ & 0,01 & 16,91 & 7,98 & 5,95 & 2,25 \\
\hline \multirow[t]{3}{*}{ SJ } & & & 0,09 & $<0,005$ & 0,01 & $<0,005$ & 0,01 & 0,01 & 0,01 & 17,91 & 36 & 6,19 & 2,49 \\
\hline & Médi & & & & & $<0,0$ & & & 0,01 & 17,69 & & 6,00 & 2,38 \\
\hline & DP & 0,00 & 0,01 & & 0,00 & & 0,00 & 0,00 & 0,00 & 0,39 & 0,14 & 0,09 & 0,12 \\
\hline & & & & 0,01 & $<0,005$ & 0,04 & $<0,00$ & $<0,005$ & $<0,005$ & 3,75 & $34, c$ & 0,96 & 2,25 \\
\hline \multirow[t]{4}{*}{ YC } & & & & 0,02 & $<0,005$ & & $<0,00$ & $<0,005$ & $<0,005$ & 85 & & 1,06 & 2,88 \\
\hline & Médi & 55 & & 0, & & 0,04 & $<0,00$ & $<0,005$ & & 3,80 & & 1,01 & 2,64 \\
\hline & DP & 0,04 & & 0,01 & & 0,00 & & & & 0,05 & 0 , & 0,04 & 0,30 \\
\hline & & & & & & $<0,005$ & & $<0,005$ & & 11,48 & &, 59 & 2,22 \\
\hline \multirow[t]{4}{*}{ SB } & & & & & & & & & & & & 7,19 & 2,95 \\
\hline & & & & & & $<0,005$ & 0 , & $<0,005$ & $<0,005$ & 16,71 & 11,09 & 6,13 & 2,51 \\
\hline & & & & & & & & & & & & 0,80 & 0,33 \\
\hline & & & & & & & & $<0,005$ & 0,005 & 16,17 & & 3,12 & 1,20 \\
\hline \multirow[t]{4}{*}{ AS } & & & & & & & & & & 17,95 & & 10 & 1,51 \\
\hline & Méc & 0, & & & & $<0,005$ & 0, & $<0,005$ & $<0,000$ & 17,22 & 86 & 8,55 & 1,27 \\
\hline & & & & & & & & & & & & 0,45 & 0,12 \\
\hline & & 0, & & $<0,005$ & $<0,005$ & & & 0,01 & 0,005 & & & 3,56 & 2,89 \\
\hline \multirow[t]{4}{*}{ L3 } & & & & & & & & $0, c$ & $<0,005$ & & & 5,0 & 3,27 \\
\hline & & & & & $<0,005$ & $<0,005$ & & 0,01 & $<0,005$ & 10,31 & 75 & 4,53 & 3,14 \\
\hline & & & & & & & & 0,00 & & 1, & & 0,73 & 0,19 \\
\hline & & & & & & & & $<0,005$ & 0,01 & 27,97 & 36 , & 8,75 & 1,72 \\
\hline \multirow[t]{4}{*}{ SP } & & & & & & & & & 0, & 28,35 & & 97 & 1,74 \\
\hline & & & & & & & & $<$ & 0 , & 28,16 & 36 , & 86 & 1,73 \\
\hline & D & & & & & & 0,01 & & 0,00 & & & 13 & 0,01 \\
\hline & & & & & $<0,005$ & & $<0,0$ & $<0,005$ & $<0,005$ & & & 1,29 & 1,82 \\
\hline \multirow[t]{3}{*}{ NS } & & & & & & & & & &,$/ 4$ & 33 & 43 & 1,83 \\
\hline & & & & & $<0,00$ & & & & 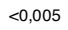 & 3,55 & 93 & 1,38 & 1,82 \\
\hline & DP & 0,01 & & & & & & & & 0,33 & 0,35 & 0,08 & 0,01 \\
\hline
\end{tabular}

As águas provenientes das fontes NS, YC, L3 e IB apresentaram teores baixos de dureza, sendo classificadas como água mole de acordo com a escala francesa $(0$ a 60mg L-1 $\mathrm{CaCO}_{3}$ ) [8]. As amostras das fontes AS, SB e SJ apresentaram teores de dureza maiores que $60 \mathrm{mg} \mathrm{L}^{-1}$ e, portanto, são classificadas como moderadamente duras (60 a $120 \mathrm{mg} \mathrm{L}^{-1} \mathrm{CaCO}_{3}$ ). A amostra que apresentou os resultados mais elevados foi a da fonte SP com teores de até $136 \mathrm{mg} \mathrm{L}^{-1}$, sendo classificada com água dura (120 a $180 \mathrm{mg} \mathrm{L}^{-1} \mathrm{CaCO}_{3}$ ).

Os resultados encontrados para a análise de dureza foram confirmados pelos valores obtidos de cálcio e magnésio, sendo que a água da fonte SP apresentou os níveis elevados de cálcio (de 27,97 a 28,35 $\mathrm{mg} \mathrm{L}^{-1}$ ) e de magnésio (de 8,75 a $8,97 \mathrm{mg} \mathrm{L}^{-1}$ ). Niveis altos de magnésio (entre 8,12 e 9,10mg L $\mathrm{m}^{-1}$ ) também foram encontrados na água AS, porém, esta fonte apresentou teores baixos de cálcio $\left(16,17\right.$ a $\left.17,95 \mathrm{mg} \mathrm{L}^{-1}\right)$, já a água IB apresentou os menores teores de cálcio $\left(0,53\right.$ a $\left.0,62 \mathrm{mg} \mathrm{L}^{-1}\right)$ e magnésio $\left(0,00\right.$ a $\left.0,02 \mathrm{mg} \mathrm{L}^{-1}\right)$.

A água IB apresentou niveis elevados de sódio $(93,61$ a $97,29 \mathrm{mg} \mathrm{L}^{-1}$ ), esses niveis podem ser considerados altos para pessoas que tenham uma dieta com restrição a este elemento (como aquelas que sofrem de problemas renais, hipertensão, etc.), para os quais o teor recomendado de sódio na água utilizada, tanto para bebida como para preparo de alimentos, é de apenas $20 \mathrm{mg} \mathrm{L}^{-1}$ [2].

As águas YC e SP também apresentaram niveis altos de sódio $\left(34,08\right.$ a 35,75 e 36,42 a $36,61 \mathrm{mg} \mathrm{L}^{-1}$, respectivamente) e a AS apresentou os menores niveis de sódio $\left(0,53\right.$ a $\left.1,24 \mathrm{mg} \mathrm{L}^{-1}\right)$ na primeira e última época de avaliação sendo que na segunda época observou-se um valor de $13,26 \mathrm{mg} \mathrm{L}^{-1}$.

Todas as amostras analisadas apresentaram teores de bário inferiores ao máximo estipulado pela legislação brasileira que é de $1 \mathrm{mg} \mathrm{L}^{-1}$ [4]. Na água IB não foi detectada a presença do metal e os maiores niveis foram encontrados na água $\operatorname{SP}\left(0,26 \mathrm{mg} \mathrm{L}^{-1}\right)$.

Não foi detectada a presença de sulfato na maioria das amostras, sendo que a água com maior teor desse ânion foi a IB com niveis entre 44,67 e 55,67 $\mathrm{mg} \mathrm{L}^{-1}$.

As maiores concentrações de cloreto foram encontradas nas águas SP, com teores próximos a $23,5 \mathrm{mg} \mathrm{L}^{-1}$. Esse valor está muito abaixo dos que a Organização Mundial de Saúde apresenta como niveis máximos desejável e permissivel, respectivamente $200 \mathrm{mg} \mathrm{L}^{-1}$ e $600 \mathrm{mg} \mathrm{L}^{-1}$ de cloreto [2].

No caso do sulfeto, as amostras SB, SP, IB e L3 apresentaram niveis muito baixos entre 0,001 e $0,002 \mathrm{mg} \mathrm{L}^{-1}$, nas demais não foi detectada a presença desse íon.

Baixos niveis de zinco (máximo de $0,03 \mathrm{mg} \mathrm{L}^{-1}$ ) foram encontrados nas águas SP, AS, SB, IB, SP e L3. Esses niveis estão abaixo dos que provocam alteração perceptivel de sabor que é de $4,3 \mathrm{mg} \mathrm{L}^{-1}$ [2].

$\mathrm{Na}$ análise de cor, as amostras NS, AS, SB, IB e SJ apresentaram resultados acima do recomendado de 5 unidades PtCo [4]. Porém, todas as amostras estavam dentro dos padrões para turbidez, confirmando o aspecto limpido que apresentavam na análise visual. Não foi observado nenhum odor fora do característico. 
As amostras SJ e L3 apresentaram teores baixos de cromo (valores máximos de $0,01 \mathrm{mg} \mathrm{L}^{-1}$ ) nas duas últimas épocas de análises. Já com relação ao chumbo, todas as amostras apresentaram baixos niveis (não detectado e máximo de $0,01 \mathrm{mg} \mathrm{L}^{-1}$ ), os quais são abaixo do limite máximo permitido pela legislação $\left(0,05 \mathrm{mg} \mathrm{L}^{-1}\right)$ [4].

Não foi detectada a presença de cobalto, cobre e cádmio em nenhuma das amostras analisadas.

Nas análises de fluoreto, realizadas na terceira época, a amostra com resultados mais altos foi a YC com $0,80 \mathrm{mg} \mathrm{L}^{-1}$ sendo que o máximo permitido pela legislação é de $1,00 \mathrm{mg} \mathrm{L}^{-1}$ [4]. Os resultados obtidos nas análises confirmaram a classificação de água fluoretada quando esta estava presente no rótulo.

\section{4 - CONCLUSÕES}

As amostras de águas minerais analisadas apresentaram composição química dentro dos limites permitidos pela legislação brasileira com algumas exceções: 4 amostras com resultados na determinação de cor acima de 5 unidades PtCo, uma amostra com $\mathrm{pH}$ fora da faixa recomendada e uma amostra com alcalinidade em hidróxidos. Os resultados obtidos mostraram que as águas minerais das oito marcas comercializadas na região de Campinas, Estado de São Paulo, são adequadas para o consumo da população, em relação ao teor dos metais presentes nas mesmas.

As águas analisadas mostraram ser predominantemente fonte de oligoelementos, pois não apresentaram nenhum ion com teores que pudessem ser incluídas em uma classificação especial.

\section{5 - REFERÊNCIAS BIBLIOGRÁFICAS}

[1] ABINAM/DNPM - ASSOCIAÇÃO BRASILEIRA DA INDÚSTRIA DE ÁGUAS MINERAIS E DEPARTAMENTO NACIONAL DE PRODUÇÃO MINERAL, Água mineral no Brasil: retrato histórico da indústria engarrafadora. São Paulo: Arte \& Ciência, 1996.

[2] BATTALHA, B.L.; PARLATORE, A.C. Controle da qualidade da água para consumo humano: bases conceituais $\mathrm{e}$ operacionais. São Paulo: CETESB, 1977. p. 138.
[3] BEAN, E.L. Potable water: quality goals. J. Am. Water Works Assoc., p. 221-330, 1974.

[4] BRASIL. Ministério da Saúde. Agência Nacional de Vigilância Sanitária. Resolução RDC no 54/2000. Diário Oficial, 15 de junho de 2000.

[5] BRASIL. Ministério da Saúde. Comissão Nacional de Normas e Padrões (C.N.N.P.A.). Resolução no 25/76, Diário Oficial, 03 de fevereiro de 1977.

[6] CARDONE, M.J. Detection and determination of error in analytical methodology. Part I. In the method verification program. J. Assoc. Anal. Chem., v. 66, n. 5, p. 1257 1282, 1983.

[7] CUNNIF, P. (ed). Official Methods of Analysis of the AOAC INTERNATIONAL. 16ed. Washington, DC: AOAC, 1995, chapter 9, p. 34.

[8] DR/2000 Spectrophotometer Handbook. 8ed. Loveland: Hach Company, 1993. 281p.

[9] HIRATA. R. Qualidade das águas subterrâneas e das águas minerais. In: SIMPÓSIO DE ÁGUAS SUBTERRÂNEAS, 1995, São Paulo. A alternativa eficaz para o suprimento de água industrial no presente e no futuro. Anais... São Paulo, 1995.

[10] LARA, A.B.W.; NAZÁRIO, G.; ALMEIDA, M.E.W.; PREGNOLATO, W. Normas Analíticas do Instituto Adolfo Lutz: métodos químicos e físicos para análise de alimentos. 3ed. São Paulo: Instituto Adolfo Lutz, 1985. p. 307-310.

[11] MERCK. Análisis de Águas. Darmstadt: E. Merck, 1981, p. 226.

[12] NISHIHARA, L.; ALABURDA, J.; MAIO, F.D. Características físico-químicas das águas de fontes minerais da região da grande São Paulo. Revista Instituto Adolfo Lutz, v. 57, n. 2, p. 19-25, 1998.

[13] PEDRO, N.A.R.; FREITAS, V.P.S.; BADOLATO, I.C.B.; OLIVEIRA, E. Determinação de metais em águas minerais da região de Campinas, São Paulo. Química Nova, v. 14, n. 4, p. 108-109, 1991.

[14] SIMÕES, R.A.P. Análise do mercado de águas minerais e suas tendências. Engarrafador Moderno, p. 13-26, 1997.

\section{6 - AGRADECIMENTOS}

Ao CNPq pelo auxílio concedido. 